\title{
Machine Learning for Turning Optical Fiber Specklegram Sensor into a Spatially-Resolved Sensing System. Proof of Concept
}

\author{
A. Rodríguez-Cuevas, M. Fontana, L. Rodríguez-Cobo, M. Lomer, and J.M. López-Higuera
}

\begin{abstract}
Fiber Specklegram Sensors (FSSs) are highly sensitive to external perturbations, however, trying to locate perturbation's position remains as a barely addressed study. In this work a system able to classify perturbations according to the place they have been caused along a multimode optical fiber has been design. As proof of concept, a multimode optical fiber has been perturbated in different points, recording the videos of the perturbations in the speckle pattern, processing these videos, training with them a machine learning algorithm and classifying further perturbations based on the spatial locations they were generated. The results show classifications up to $99 \%$ when the system has to categorize among three different locations lowering to $71 \%$ when the locations rise to ten.
\end{abstract}

Index Terms-Fiber optic sensors, Multimode waveguides, Neural networks, Pattern recognition, Speckle, Speckle Interferometry.

\section{INTRODUCTION}

$\mathrm{T}$ he importance of the optical fiber sensors has been growing strongly in the last decades since their study started more than forty years ago. Among different applications, strain and temperature sensing has dominated the market and still attracts a huge interest [1]. However, apart from these typical applications, optical fiber sensors can be employed for many different purposes. In particular, the use of interferometers can provide exceptional vibration-sensing capabilities. Interferometric optical fiber sensors, like the fiber specklegram sensor (FSSs), exhibit remarkably good sensitivity to external vibrations which can be used as a sensing tool for applications such as non-contact patient monitoring $[2,3]$ or surveillance $[4$, 5]. The speckle phenomenon in optical fibers was described by Spillman et al. who first realized about its utility as a sensing tool [6]. However, FSSs measure the perturbation by integrating all the small contributions at each different location, making much more complicated to locate each specific perturbation. This means that, when a speckle system in optical

This work has been supported by the Spanish Government through the Ministry of Economy and Competitiveness project TEC2016-76021-C2-2-R (AEI/FEDER, UE).

A. Rodriguez-Cuevas is with Grupo de Ingeniería Fotónica, Universidad de Cantabria, Santander, Spain

(e-mail: alberto.rodriguezc@alumnos.unican.es)

M. Fontana is with Department of Information Engineering, Università degli Studi di Padova, Italy (e-mail: marco.fontana.5@studenti.unipd.it) fibers is used for sensing purposes, it measures the perturbations generated all along the fiber as a "unique sensing point", independently of where the perturbation is being generated.

\section{A. Speckle Phenomenon in Optical Fiber}

The speckle phenomenon occurs when coherent light is conducted towards a multimode optical fiber. At the other end of the fiber, a granular pattern is generated, called specklegram [6]. This specklegram (speckle pattern) is highly sensitive to external perturbations such as vibrations, temperature, or strain [1-6]. A multimode optical fiber carries many different optical paths. In a simple way, these optical paths can be understood as the different paths light follows when travelling along the fiber, and are known as modes. Due to the different length of the modes, the optical phase of every light beam differs from each other, resulting in interference effects at the end of the optical fiber. In that sense, if the phase delay is $2 \pi$ radians (or multiples), the interference phenomenon is constructive. In the opposite case, if the phase delay is $\pi$ radians, the interference phenomenon is destructive, and therefore the intensities of each light beam are subtracted. All the other possible phase delays produce intermediate interferences being partially destructive or partially constructive. Eventually, all these interferences generate a light pattern at the other end of the fiber, which is composed of brighter and darker dots known as speckles.

Indeed, speckle phenomenon in fiber optic is so highly nonlinear that obtaining a detailed mathematical model able to relate the behavior of the modes and the position of the perturbation would be too complex and would fail to provide much of the insight. Furthermore, most of the systems using the speckle phenomenon as sensing tool are focused on how much the speckle pattern varies in a global sense, addressing the sensor response from a statistical perspective [2]. In fact, the current state-of-the-art indicates there are almost no publications about the speckle system in fiber optic used as a distributed sensing method [7, 8]. Nevertheless, Plöchner M. et al. [9] proved that multimode fibers might not be as

L. Rodríguez-Cobo is with Centro de Investigación Biomédica en Red en Bioingeniría, Biomateriales y Nanomedicina (CIBER-BBN), Cantabria, Spain (e-mail: luis.rodriguez@unican.es)

M. Lomer and J.M. López Higuera is with Grupo de Ingeniería Fotónica, Universidad de Cantabria, Santander, Spain. Centro de Investigación Biomédica en Red en Bioingeniría, Biomateriales y Nanomedicina (CIBERBBN), Cantabria, Spain. Instituto de Investigación Sanitaria Valdecilla (IDIVAL), Cantabria, Spain (e-mail: matias.lomer@unican.es; miguel.lopezhiguera@unican.es) 
unpredictable as it was previously thought by studying its behaviors in rather small segments of fiber optic. Some other authors have performed similar studies as well [10 - 12]. Moreover, the synthesis of the desired intensity profile at the output of the multimode fiber has led some authors to obtain some degree of deterministic transmission algorithms $[12,13]$.

\section{B. Hypothesis and Purpose}

The starting point of this hypothesis is provided by previous works already cited. These works suggest that, in a way, getting to a degree of spatial-resolved sensing system using specklegram sensors in fiber optic could be possible. Although the propagation of light waves in dispersive media (such as multimode fibers) has a very large number of degrees of freedom, the location of the perturbation along the fiber might have a consistent effect on the changes of the speckle pattern. In addition, even though the speckle phenomenon in fiber optic can result in a very complex model, the knowledge gathered so far about this effect and how the disorder affects light transport in crystalline lattice [14] supply even more evidences. Indeed, all of this can lead to think the way the different modes vary and exchange energy among them could be the same. On the assumption that we could generate the exact same perturbation on the exact point in an ideal system.

Therefore, the purpose of this work is to find out whether there is a clear correlation between the distance at which the perturbation is generated in a fiber optic and the changes that occur in the outer speckle pattern. In this regard, if this hypothesis is confirmed a new set of applications with specklegram sensors could be developed. We do not attempt here to provide a detailed theoretical study of the way the modes vary in relation with the distance where the perturbation is caused. Rather, the aim is to provide a proof-of-concept able to show the capabilities of machine learning techniques as a monitoring system for a spatial-resolved FSSs sensor.

\section{Set Up ANd PRocessing Method}

In order to validate this hypothesis, it was decided to use an approach based on machine learning techniques. Thus, by generating very big amounts of perturbations in the same places of a multimode fiber, recording the videos of the changes in the speckle pattern, processing them and training with them different machine learning algorithms, it could be possible to predict the point where new perturbations were being generated along the fiber.

Eventually, two different systems were created: the first could distinguish among three different locations, while the second one was able to distinguish among 10 different sections.

\section{A. Set Up}

The main objective to consider for designing this set up was to generate a system capable of perturbing the fiber at different locations, but with the highest degree of similarity between the perturbations caused on them. It was critically needed that the machine learning techniques did not classify based on the mechanical differences in the perturbation, but instead classified the videos based on how the specklegram changes, depending on the point where the perturbation was generated.
Following this previous consideration, two equivalent systems were designed and constructed. The only difference between them was the first one consisted of a 6-meters-long plastic optical fiber, for generating perturbations in the three points, while the second one consisted of a 12-meters-long plastic optical fiber, for generating perturbations in 10 spatially distributed points. The rest of the elements were the same for both systems: a CCD camera, a coherent light source $(638 \mathrm{~nm}$ wavelength), and a linear stepper motor. This motor had an extension coupled which acted as a hitter perturbing the fiber in different points, as it is shown in the schema of Figure 1.

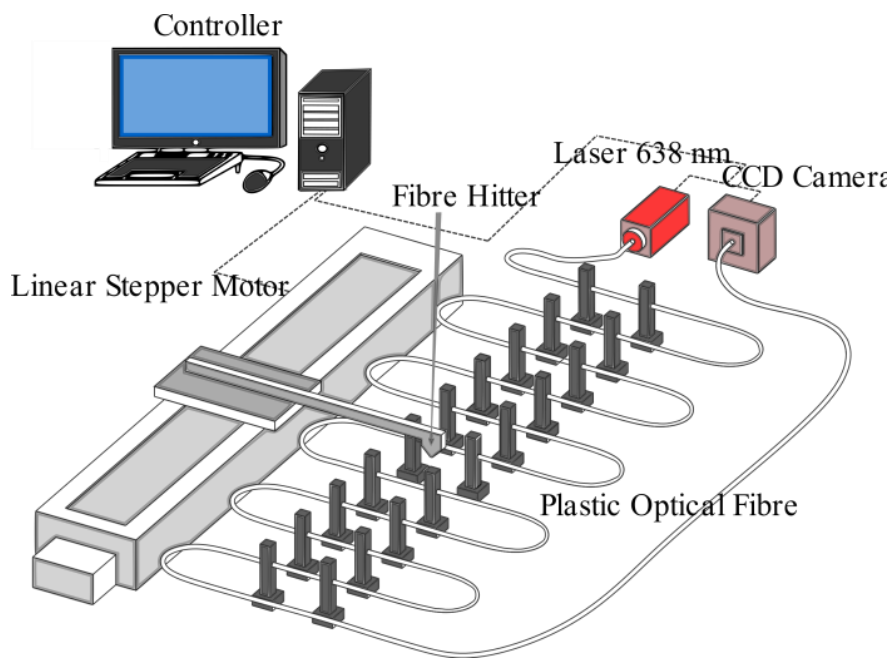

Fig. 1. Set up used for the automatic recording of videos of the speckle pattern while perturbating a multimode plastic optical fiber at 10 different locations.

The system was design in a way that every perturbation was lasting slightly less than a third of second. The frame rate of the sensing element was 30 frames per second. So, from every perturbation a nine-frames color video was recorded. Additionally, some other videos of tactile perturbations were recorded too. In these videos the perturbations were done by touching the fiber with short sticks or just the fingertip. All these short videos were processed, normalized and labelled.

Since hundreds of thousands of videos were recorded in different days, each group of videos captured sequentially was considered a dataset, and when the capture was stopped for some time and then resumed, a new dataset was generated. This fact was rather important because it was observed in some preliminary test that the categorization accuracy when using data of the same dataset (same conditions) tend to be better than when using mixed data from different datasets. In the results section this fact will be discussed in more detail.

\section{B. Data Preparation}

All the recorded videos were stored on a hard drive until the dataset was complete. Once the record was finished, the videos were pre-processed for subtracting only the useful information. After that, each video was converted into a single image, which was then normalized and classified.

Every video was converted to a grayscale, then, a differential processing was done by subtracting one frame from the previous one (see Figure 2.b), obtaining a video sequence in 
which each frame has only its variation to the previous one pixel by pixel. Each pixel $\left(\mathrm{p}_{\mathrm{n}, \mathrm{m}}\right)$ of a differentially processed $\mathrm{i}$-th frame, Diff_Frame $\mathrm{p}_{\mathrm{n}, \mathrm{m}}^{\mathrm{i}}$ for two specklegrams of NxM pixels can be defined as follows:

$$
\text { Diff_Frame } e_{p_{n, m}}^{i}=\mid \text { Frame }_{p_{n, m}}^{i}-\text { Frame }_{p_{n, m}}^{i-1} \mid
$$

where Frame ${ }_{p_{n, m}}^{i}$ corresponds to the pixel of the $n, m$ position of the specklegram frame $i$.

In this way, the pixels of each image have information about the speckles which are changing and not about the ones which are not. In last two steps, the differential frames associated to each perturbation hit (h), comprised within an averaging window of nine frames $(\mathrm{Nw}=4)$ were summed into a single image (see Figure 2.c), and this image was pondered by dividing the value of every pixel by the sum of the values of all pixels in the image Thus, each pixel $\left(\mathrm{p}_{\mathrm{n}, \mathrm{m}}\right)$ of the averaged frame associated at each hit (h) can be computed as:

$$
\text { Frame_sum }_{\mathrm{p}_{\mathrm{n}, \mathrm{m}}}^{\mathrm{h}}=\sum_{\mathrm{i}=\mathrm{h}-\mathrm{Nw}}^{\mathrm{h}+\mathrm{Nw}} \text { Diff_Frame }_{\mathrm{p}_{\mathrm{n}, \mathrm{m}}}^{\mathrm{i}}
$$

After computing the averaged Frame_sum $\{\mathrm{h}\}$ it is normalized (Frame_norm $\{\mathrm{h}\}$ ) by the intensity sum of every pixel comprised within the frame. The fundamental reason of this pre-processing method is making sure the obtained classification is based on the changes generated in the pattern. In particular, the aim is to avoid a categorization based on temporal or intensity patterns. In this way, any degree of classification achieved by the machine learning techniques would be obtained based on the differences in the pattern behavior derived from the place the perturbations were caused.

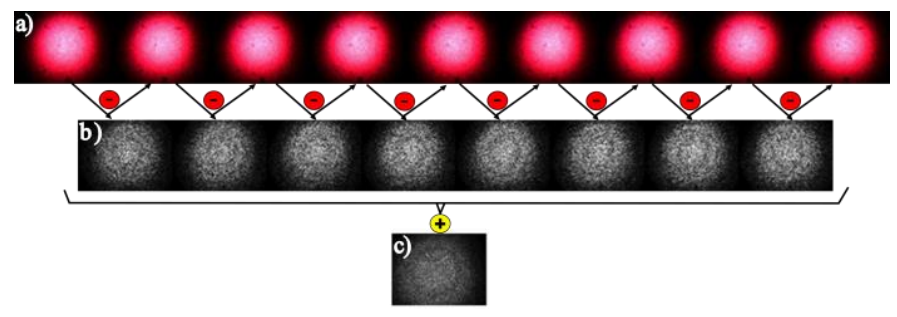

Fig. 2. a) Frames related to a detected perturbation in $240 \mu \mathrm{m}$ optical fiber; b) Differential frames of the ones in Fig 2.a; c) Example of the result of the sum and the normalization of the nine differential frames.

\section{Machine Learning}

Two different supervised machine-learning techniques were selected and implemented to predict where the perturbations were being caused. In both cases the algorithms were implemented in Matlab R2016a. The first approach, employed as a reference, was based on the training of a feedforward neural network, by means of the images previously normalized and categorized. There are evidences of good outcomes when using this kind of algorithms for processing speckle images $[15,16]$. This network consisted of three layers of neurons of different sizes, related one to the other as follows (see Figure 3.a). The first layer had a number of neurons equal to the number of pixels, while the second layer had a dimension between 10 and 20 neurons. The third layer was composed of a number of neurons equal to the number of categories we aim to characterize ( 3 in the Experiment 1 and 10 in the Experiment 2).

The second approach was based on a Convolutional Neural Network $(\mathrm{CNN})$ that provided a structure composed of twelve layers. The input layer (imageInputLayer) was followed by two sequences of convolution, max pooling and rectifier linear unit (ReLU) layers and after them there were fully connected layer, ReLu, Fully Connected Layer, Softmax and finally the classification layer. This last one was the output layer which computes the class and scores in the classification. In Figure 3.b. a simplification of the structure can be noticed. This method has been chosen based on its accuracy for classifying images [17]. Once again, the trained network obtained with this procedure provided the classification of a given input as the maximum of the values associated with the related output neurons.

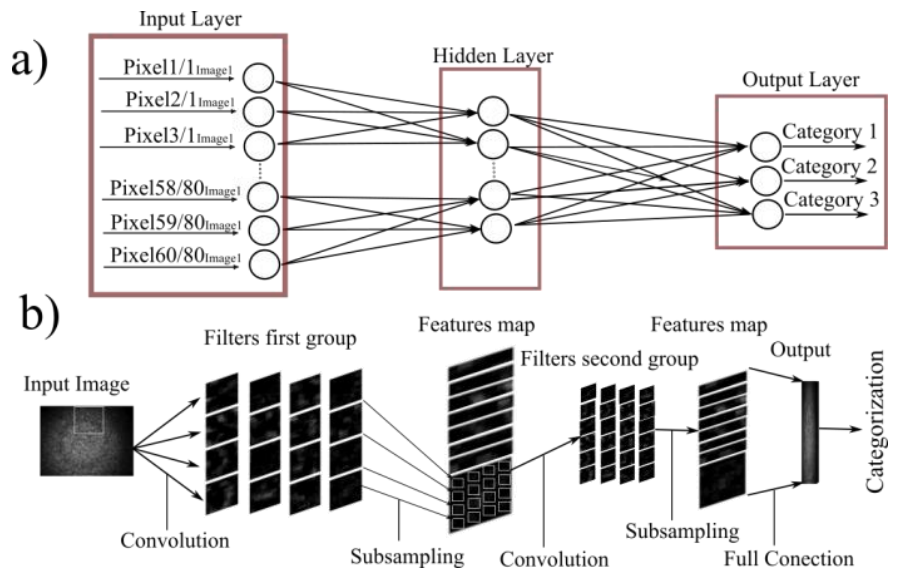

Fig. 3. a) Inputs and outputs of a neuron in the network; b) Structure of the convolutional neural network.

\section{RESULTS}

Initially, a bunch of experiments was carried out for checking the availability of the process. In these experiments, different kinds of pre-processing methods, video resolution, spatial configuration, machine learning algorithms and size of the datasets were tested. After multiple cross combinations and having recorded hundreds of thousand videos in different conditions, two different fiber optics were selected: 50 and 240 $\mu \mathrm{m}$. The resolution of the images was 60 by 80 pixels, and the distance between the perturbed points was two meters long. Each configuration was tested using two different machine learning methods. In Figure 4, the confusion matrices of the selected combinations are displayed.

This confusion matrix represents the percentage of correct classification of the data that the algorithm has categorized. 


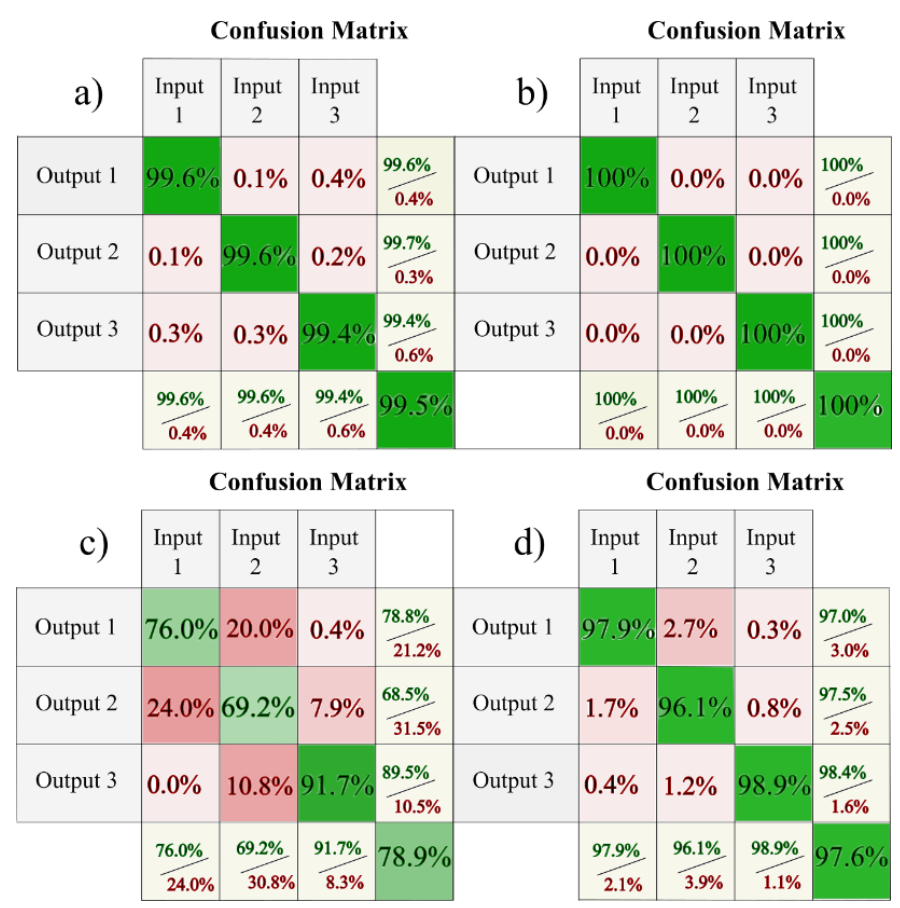

Fig. 4. a) Confusion matrix $50 \mu \mathrm{m}$ fiber and convolutional neural network; b) Confusion matrix $50 \mu \mathrm{m}$ fiber and feedforward neural network. c) Confusion matrix $240 \mu \mathrm{m}$ fiber and convolutional neural network. d) Confusion matrix $240 \mu \mathrm{m}$ fiber and feedforward neural network.

These initial results show some remarkable degrees of classification when the algorithms classify videos which are not part of the training set. Besides, it is clear that the $50 \mu \mathrm{m}$ fiber achieves better classifications than the fiber of $240 \mu \mathrm{m}$, while the feedforward neural network usually offers better degrees of classifications than the convolutional neural network. After ensuring there was some degree of classification of the videos based on the place where the perturbation was being caused, a larger test dataset was carried out in Experiment 1.

\section{A. Experiment I}

In the first experiment, the fiber was perturbed only in three points. The results are divided into two different tables. In the first one (Table 1), all the datasets are mixed, that means that there are videos of the same datasets for testing and training. From this mix of videos, the $75 \%$ is used for training and the remaining $25 \%$ is used for testing. However, the second table (Table 2) exhibits the results when the data sets for testing and training are different from one another. The classifications displayed in the tables correspond to the total degree of classification.

Table 1. Results of the overall accuracy when using parts of the same datasets for training and for testing. The total number of videos of used for training and testing is represented while the letters following it represent the perturbation method: linear stepper $[\mathrm{L}]$ or manual $[\mathrm{M}]$.

\begin{tabular}{ccccc}
\hline Fiber & Train Data & Test Data & $\begin{array}{c}\text { Feedforward } \\
\text { Neural Net. }\end{array}$ & $\begin{array}{c}\text { Convolutional } \\
\text { Neural Net. }\end{array}$ \\
\hline $\mathbf{5 0}$ & $112,500[\mathrm{~L}]$ & $37,500[\mathrm{~L}]$ & $99.8 \%$ & $99.71 \%$ \\
$\boldsymbol{\mu m}$ & $17,050[\mathrm{M}]$ & $5,680[\mathrm{M}]$ & $79.1 \%$ & $78.3 \%$ \\
$\mathbf{2 4 0}$ & $43,020[\mathrm{~L}]$ & $14,340[\mathrm{~L}]$ & $79.1 \%$ & $80.08 \%$ \\
$\boldsymbol{\mu m}$ & $14,550[\mathrm{M}]$ & $4,850[\mathrm{M}]$ & $78.7 \%$ & $72.26 \%$ \\
\hline
\end{tabular}

Table 2. Results of the overall accuracy when using different datasets for training than testing. The total number of videos used for training and testing is represented while the letters following it represent the perturbation method: linear stepper [L] or manual [M].

\begin{tabular}{ccccc}
\hline Fiber & Train Data & Test Data & $\begin{array}{c}\text { Feedforward } \\
\text { Neural Net. }\end{array}$ & $\begin{array}{c}\text { Convolutional } \\
\text { Neural Net. }\end{array}$ \\
\hline $\mathbf{5 0}$ & $112,500[\mathrm{~L}]$ & $37,500[\mathrm{~L}]$ & $96.9 \%$ & $90.34 \%$ \\
$\mathbf{\mu m}$ & $17,050[\mathrm{M}]$ & $5,680[\mathrm{M}]$ & $53.4 \%$ & $48.91 \%$ \\
$\mathbf{2 4 0}$ & $43,020[\mathrm{~L}]$ & $14,340[\mathrm{~L}]$ & $73.7 \%$ & $71.01 \%$ \\
$\boldsymbol{\mu m}$ & $14,550[\mathrm{M}]$ & $4,850[\mathrm{M}]$ & $75.6 \%$ & $66.07 \%$ \\
\hline
\end{tabular}

As it is shown in both tables, classifications are very precise over $99 \%$ when the $50 \mu \mathrm{m}$ plastic optical fiber is used, while only $80 \%$ accuracy is obtained when using a $240 \mu \mathrm{m}$ plastic optical fiber. Due to its bigger diameter, the number of modes generated in a $240 \mu \mathrm{m}$ fiber is several times greater than the number of modes in a $50 \mu \mathrm{m}$ fiber. Since the complexity of the system is fully proportional to the number of modes propagated by the fiber, the size of the dataset required might need to be significantly larger in the 240 fiber. Furthermore, the camera resolution might have to be higher as well, since the speckles are smaller.

When analyzing the results of the perturbations caused manually, in every case the classifications are less precise, probably because the variations introduced manually have a wider deviation, making their classification more challenging. Furthermore, the differences become clearer when different datasets are used. Comparing the results of the handmade perturbations, the differences between the datasets are more important because the way the fiber was perturbed was different from dataset to dataset, so it seems logical the classification is worse than the one of the combined datasets.

Finally, both machine learning methods are rather accurate, although the feedforward neural network method is slightly more precise.

\section{B. Experiment II}

Based on the results obtained in the first experiment, we decided to increase the complexity of the system by increasing the number of points where the fiber was perturbed. However, in this case only the worst behaving fiber was selected, i.e. $240 \mu \mathrm{m}$ fiber. In this test, a set up for perturbing the fiber at 10 different points was prepared, being the distances between points one-meter-long, as it can be seen in Figure 1. The preprocessing of every video was the same than the one done in the previous experiment. However, in this experiment, the conditions remained unchanged, and the system was not modified in any way at any moment during the whole recording time. Therefore, the training and testing videos belong to the same dataset.

Figure 5 displays the classifications achieved using the feedforward neural network and convolutional neural network techniques. Once again, it can be seen that the system is able to classify videos of perturbations which are not part of the training set. The categories are in a sequential order, being Input 
1 the point closer to the laser and Input 10 the point closer to the camera.

\section{Confusion Matrix}

\begin{tabular}{|c|c|c|c|c|c|c|c|c|c|c|c|}
\hline a) & $\begin{array}{c}\text { Input } \\
1\end{array}$ & $\begin{array}{c}\text { Input } \\
2\end{array}$ & $\begin{array}{c}\text { Input } \\
3\end{array}$ & $\begin{array}{c}\text { Input } \\
4\end{array}$ & $\begin{array}{c}\text { Input } \\
5\end{array}$ & $\begin{array}{c}\text { Input } \\
6\end{array}$ & $\begin{array}{c}\text { Input } \\
7\end{array}$ & $\begin{array}{c}\text { Input } \\
8\end{array}$ & $\begin{array}{c}\text { Input } \\
9\end{array}$ & $\begin{array}{c}\text { Input } \\
10\end{array}$ & \\
\hline Output 1 & $44.9 \%$ & $35.9 \%$ & $12.6 \%$ & $6.3 \%$ & $0.0 \%$ & $0.5 \%$ & $0.0 \%$ & $0.0 \%$ & $0.0 \%$ & $0.0 \%$ & $\frac{44.8 \%}{55.19 \%}$ \\
\hline Output 2 & $31.2 \%$ & $38.6 \%$ & $6.2 \%$ & $20.4 \%$ & $1.0 \%$ & $1.9 \%$ & $0.6 \%$ & $0.0 \%$ & $0.0 \%$ & $0.0 \%$ & $\frac{38.64 \%}{61.36 \%}$ \\
\hline Output 3 & $17.3 \%$ & $10.8 \%$ & $67.7 \%$ & $1.2 \%$ & $0.0 \%$ & $0.1 \%$ & $2.7 \%$ & $0.0 \%$ & $0.0 \%$ & $0.0 \%$ & $\frac{67.84 \%}{32.16 \%}$ \\
\hline Output 4 & $8.7 \%$ & $16.6 \%$ & $0.7 \%$ & $41.9 \%$ & $13.3 \%$ & $16.5 \%$ & $0.6 \%$ & $1.5 \%$ & $0.0 \%$ & $0.0 \%$ & $\frac{42.0 \%}{58.0 \%}$ \\
\hline Output 5 & $0.1 \%$ & $0.2 \%$ & $0.0 \%$ & $9.3 \%$ & $45.5 \%$ & $28.5 \%$ & $0.0 \%$ & $16.1 \%$ & $0.2 \%$ & $0.6 \%$ & $\frac{44.0 \%}{56.0 \%}$ \\
\hline Output 6 & $0.6 \%$ & $1.2 \%$ & $0.0 \%$ & $15.0 \%$ & $30.9 \%$ & $649.7 \%$ & $0.0 \%$ & $2.7 \%$ & $0.0 \%$ & $0.0 \%$ & $\frac{49.7 \%}{50.3 \%}$ \\
\hline Output 7 & $0.8 \%$ & $1.2 \%$ & $2.2 \%$ & $1.8 \%$ & $3.5 \%$ & $0.0 \%$ & $89.8 \%$ & $2.7 \%$ & $1.1 \%$ & $0.0 \%$ & $\frac{87.1 \%}{12.9 \%}$ \\
\hline Output 8 & $0.0 \%$ & $0.0 \%$ & $0.0 \%$ & $1.8 \%$ & $12.0 \%$ & $4.9 \%$ & $3.3 \%$ & $61.9 \%$ & $16.1 \%$ & $0.0 \%$ & $\frac{61.9 \%}{38.1 \%}$ \\
\hline Output 9 & $0.0 \%$ & $0.0 \%$ & $0.0 \%$ & $0.0 \%$ & $0.0 \%$ & $0.0 \%$ & $0.4 \%$ & $11.6 \%$ & $87.8 \%$ & $0.2 \%$ & $\frac{87.8 \%}{12.2 \%}$ \\
\hline \multirow[t]{2}{*}{ Output 10} & $0.0 \%$ & $0.0 \%$ & $0.0 \%$ & $0.0 \%$ & $0.0 \%$ & $0.1 \%$ & $0.1 \%$ & $0.1 \%$ & $0.2 \%$ & $99.4 \%$ & $\frac{99.5 \%}{0.05 \%}$ \\
\hline & $\frac{43.3 \%}{56.6 \%}$ & $\frac{37.0 \%}{63.0 \%}$ & $\frac{75.7 \%}{24.3 \%}$ & $\frac{42.9 \%}{57.1 \%}$ & $\frac{42.8 \%}{57.2 \%}$ & $\frac{48.3 \%}{56.6 \%}$ & $\frac{92.1 \%}{7.9 \%}$ & $\frac{64.1 \%}{35.9 \%}$ & $\frac{83.3 \%}{16,6 \%}$ & $\frac{99,2 \%}{0,8 \%}$ & $63.1 \%$ \\
\hline
\end{tabular}

Confusion Matrix

\begin{tabular}{|c|c|c|c|c|c|c|c|c|c|c|c|}
\hline b) & $\begin{array}{c}\text { Input } \\
1\end{array}$ & $\begin{array}{c}\text { Input } \\
2\end{array}$ & $\begin{array}{c}\text { Input } \\
3\end{array}$ & $\begin{array}{c}\text { Input } \\
4\end{array}$ & $\begin{array}{c}\text { Input } \\
5\end{array}$ & $\begin{array}{c}\text { Input } \\
6\end{array}$ & $\begin{array}{c}\text { Input } \\
7\end{array}$ & $\begin{array}{c}\text { Input } \\
8\end{array}$ & $\begin{array}{c}\text { Input } \\
9\end{array}$ & $\begin{array}{c}\text { Input } \\
10\end{array}$ & \\
\hline Output 1 & $66.2 \%$ & $43.1 \%$ & $23.3 \%$ & $2.6 \%$ & $0.0 \%$ & $0.0 \%$ & $0.0 \%$ & $0.0 \%$ & $0.0 \%$ & $0.0 \%$ & $\frac{48.9 \%}{51.1 \%}$ \\
\hline Output 2 & $22.7 \%$ & $33.4 \%$ & $5.9 \%$ & $4.8 \%$ & $0.0 \%$ & $0.0 \%$ & $0.0 \%$ & $0.0 \%$ & $0.0 \%$ & $0.0 \%$ & $\frac{49.8 \%}{50.2 \%}$ \\
\hline Output 3 & $5.1 \%$ & $1.6 \%$ & $69.2 \%$ & $0.0 \%$ & $0.0 \%$ & $0.0 \%$ & $10.2 \%$ & $0.0 \%$ & $0.0 \%$ & $0.0 \%$ & $\begin{array}{l}80.2 \% \\
19.8 \%\end{array}$ \\
\hline Output 4 & $0.0 \%$ & $20.8 \%$ & $0.2 \%$ & $67.8 \%$ & $4.9 \%$ & $5.4 \%$ & $0.0 \%$ & $0.4 \%$ & $0.0 \%$ & $0.0 \%$ & $\frac{64.5 \%}{35.5 \%}$ \\
\hline Output 5 & $0.2 \%$ & $0.2 \%$ & $0.0 \%$ & $11.9 \%$ & $75.1 \%$ & $58.1 \%$ & $0.0 \%$ & $5.2 \%$ & $0.0 \%$ & $0.0 \%$ & $\frac{49.7 \%}{50.3 \%}$ \\
\hline Output 6 & $0.2 \%$ & $0.5 \%$ & $0.0 \%$ & $12.6 \%$ & $12.0 \%$ & $35.1 \%$ & $0.0 \%$ & $0.0 \%$ & $0.0 \%$ & $0.0 \%$ & $\frac{58.4 \%}{41.6 \%}$ \\
\hline Output 7 & $0.0 \%$ & $0.4 \%$ & $1.1 \%$ & $0.2 \%$ & $0.0 \%$ & $0.0 \%$ & $89.7 \%$ & $0.6 \%$ & $0.0 \%$ & $0.0 \%$ & $\frac{97.4 \%}{2.6 \%}$ \\
\hline Output 8 & $0.0 \%$ & $0.0 \%$ & $0.0 \%$ & $0.2 \%$ & $8.0 \%$ & $1.5 \%$ & $0.0 \%$ & $82.3 \%$ & $6.2 \%$ & $0.0 \%$ & $\begin{array}{l}83.8 \% \\
16.2 \%\end{array}$ \\
\hline Output 9 & $0.0 \%$ & $0.0 \%$ & $0.0 \%$ & $0.0 \%$ & $0.0 \%$ & $0.0 \%$ & $0.0 \%$ & $11.5 \%$ & $93.8^{\circ}$ & $0.0 \%$ & $\frac{89.0 \%}{11.0 \%}$ \\
\hline \multirow[t]{2}{*}{ Output 10} & $0.0 \%$ & $0.0 \%$ & $0.0 \%$ & $0.0 \%$ & $0.0 \%$ & $0.0 \%$ & $0.0 \%$ & $0.0 \%$ & $0.0 \%$ & $100 \%$ & $\frac{100 \%}{0.0 \%}$ \\
\hline & $\frac{66.2 \%}{33.8 \%}$ & $\frac{33.4 \%}{66.6 \%}$ & $\begin{array}{l}\frac{69.2 \%}{30.8 \%} \\
\end{array}$ & $\frac{67.8 \%}{32.2 \%}$ & $\frac{75.1 \%}{24.9 \%}$ & $\frac{35.1 \%}{64.9 \%}$ & $\frac{89.7 \%}{10.3 \%}$ & $\frac{82.2 \%}{17.8 \%}$ & $\frac{93.8 \%}{6.2 \%}$ & $\frac{100 \%}{0,0 \%}$ & $71.3 \%$ \\
\hline
\end{tabular}

Fig. 5. a) Convolutional neural network confusion matrix for the $240 \mu \mathrm{m}$ fiber. b) Feedforward neural network confusion matrix for the $240 \mu \mathrm{m}$ fiber.

In general, the results are quite accurate, getting up to $71.3 \%$ of correct categorization. It can be seen in Figure 5 that the system tends to confuse the categories with the nearby ones, while the percentage of misclassification decrease strongly when the categories are far from each other. The relevant result of the system is the accuracy increases for those perturbations represented by the last inputs $10,9,8$. Indeed, in previous research it has been identify that perturbations in a multimode optical fiber last for a certain length along the fiber, and the farer the perturbation is done from the sensing element the weaker the perturbation appears [6]. Therefore, one of the possible reasons why there are higher degrees of classifications in perturbations closer to the camera is that they get a lower degree of noise.

On the other hand, the stability of the high and low order modes may be critical in this process, since the high-order modes in a multimode fiber are more susceptible to instabilities that break their symmetries [18]. Some other authors have proved the stability of nonlinear modes against small perturbations, showing for high-order modes, the existence of symmetry-breaking unstable modes may be observed [19].

\section{CONCLUSIONS}

Based on the results obtained in this proof of concept, a machine learning algorithm can be trained for classifying perturbations according to the distance where the perturbation is caused along a multimode optical fiber. The pre-processing method makes sure the videos are not classified for the differences in the intensity of perturbation or the differences in the temporal evolution of the perturbation. This method has been proved by the accuracy in the results of the perturbations generated manually, up to $79 \%$ of classification among three categories. However, any slight modification in the conditions of the environment or in the alignment of the fiber with the camera will generate an important degree of misclassification in the machine learning method. At the same time, when the number of points is increased up to 10 , the classification percentage decrease to $71 \%$. Furthermore, an interesting tendency is observed: the classifications are more accurate for the perturbations that have been generated closer to the sensing element. That might suggest the perturbations tend to become more diffuse as the light travels further through the fiber. Moreover, the existence of some modes more stable than others could lead to a higher degree of repeatability. In conclusion, despite the proposed system represents a very basic set up, it is able to achieve interesting results, which can be enhanced in future works implementing different machine learning techniques or focusing the analysis to some specific regions of the specklegram.

\section{REFERENCES}

1. Lee, B. (2003). Review of the present status of optical fiber sensors Optical Fiber Technology, 9(2), 57-79. doi:10.1016/S10685200(02)00527-8

2. Efendioglu, H. S. (2017). A review of fiber-optic modal modulated sensors: Specklegram and modal power distribution sensing. IEEE Sensors Journal, 17(7), 2055-2064

3. Rodriguez-Cuevas A, Pena ER, Rodriguez-Cobo L, Lomer M, Higuera JM. Low-cost fiber specklegram sensor for noncontact continuous patient monitoring. J Biomed Opt. 2017 Mar 1;22(3):37001.

4. Dhall, A., Chhabra, J. K., \& Aulakh, N. S. (2005). Intrusion detection system based on speckle pattern analysis. Experimental Techniques, 29(1), 25-31.

5. Rodriguez-Cuevas, A., Rodriguez-Cobo, L., Lomer, M., \& LopezHiguera, J. M. (2017, April). Safe and private pedestrian detection by a low-cost fiber-optic specklegram. In Optical Fiber Sensors Conference (OFS), 2017 25th (pp. 1-4). IEEE. 
6. Spillman W, Kline B, Maurice L, Fuhr P. Statistical-mode sensor for fiber optic vibration sensing uses. Appl Opt. 1989;28(15):3166-76.

7. Kotov O, Chapalo I. Mode-mode fiber interferometer with impact localization ability. SPIE Photonics Europe; International Society for Optics and Photonics; 2016.

8. Fujiwara E, Wu YT, dos Santos, Murilo Ferreira Marques, Schenkel EA, Suzuki CK. Development of a tactile sensor based on optical fiber specklegram analysis and sensor data fusion technique. Sensors and Actuators A: Physical. 2017;263:677-86.

9. Plöschner M, Tyc T, Čižmár T. Seeing through chaos in multimode fibres. Nature Photonics. 2015;9(8):529-35.

10. Popoff, S. M., Lerosey, G., Carminati, R., Fink, M., Boccara, A. C., \& Gigan, S. (2010). Measuring the transmission matrix in optics: an approach to the study and control of light propagation in disordered media. Physical review letters, 104(10), 100601.

11. Carpenter, J., Eggleton, B. J., \& Schröder, J. (2016). Complete spatiotemporal characterization and optical transfer matrix inversion of a 420 mode fiber. Optics letters, 41(23), 5580-5583.

12. Mahalati, R. N., Askarov, D., Wilde, J. P., \& Kahn, J. M. (2012). Adaptive control of input field to achieve desired output intensity profile in multimode fiber with random mode coupling. Optics express, 20(13), 14321-14337.

13. Papadopoulos, S. Farahi, C. Moser, and D. Psaltis, "Focusing and scanning light through a multimode optical fiber using digital phase conjugation," Opt. Express 20, 10583-10590 (2012).

14. Segev, M., Silberberg, Y., \& Christodoulides, D. N. (2013). Anderson localization of light. Nature Photonics, 7(3), 197-204

15. Efendioglu, H. S., Yildirim, T., \& Toker, O. (2011). Advanced image processing and artificial intelligence based approaches to fiber optic statistical mode sensor design. Paper presented at the Proceedings of SPIE - the International Society for Optical Engineering, , 7982 doi: $10.1117 / 12.880054$

16. Efendioglu, H. S., Yildirim, T., Toker, O., \& Fidanboylu, K. (2012) Design of intelligent fiber optic statistical mode sensors using novel features and artificial neural networks. Paper presented at the INISTA 2012 - International Symposium on INnovations in Intelligent SysTems and Applications, doi:10.1109/INISTA.2012.6247006

17. Liu, W., Wang, Z., Liu, X., Zeng, N., Liu, Y., \& Alsaadi, F. E. (2017). A survey of deep neural network architectures and their applications. Neurocomputing, 234, 11-26.

18. Longhi, S., \& Janner, D. (2004). Self-focusing and nonlinear periodic beams in parabolic index optical fibres. Journal of Optics B: Quantum and Semiclassical Optics, 6(5), S303.

19. Longhi, S., \& Janner, D. (2004). Self-focusing and nonlinear periodic beams in parabolic index optical fibres. Journal of Optics B: Quantum and Semiclassical Optics, 6(5), S303. 